Pecvnia, 7 (2008), pp. 25-44

\title{
El Comité de Empresa Europeo como instrumento de participación de los trabajadores a nivel supranacional
}

\author{
Henar Álvarez Cuesta \\ halvc@unileon.es \\ Universidad de León \\ Área de Derecho del Trabajo y de la Seguridad Social \\ Fac. de Ciencias del Trabajo \\ Campus de Vegazana, $\mathrm{s} / \mathrm{n}$ \\ Recibido: Octubre 2008 \\ Aceptado: Junio 2009

\begin{abstract}
24071 León (España)
\end{abstract}
La Directiva 94/45/CE del Consejo, de 22 de septiembre, traspuesta por la Ley 10/1997, de 24 de abril, trata de mejorar los derechos de información y consulta de los representantes a nivel de empresa o grupo de empresas de dimensión comunitaria y garantizar el acceso de los representantes de los trabajadores a los verdaderos centros de decisión. Los empresarios y trabajadores afectados tienen la facultad, previa una fase de negociación de constituir un organismo específico de representación (comité de empresa europeo) o fijar como mecanismo alternativo el ejercicio de los derechos de información y consulta fuera del citado comité.

Palabras clave: Comité de Empresa Europeo, acuerdo, negociación, representación.
The Directive 94/45/CE, 22 of September, transposed by Ley 10/1997, 24 of April, looks for increase the rights of information and participation of the workers. The companies and the employees can establish, first, they have to negotiation, a European Works Council or a procedure for informing and consulting employees in every Community-scale undertaking and every Community-scale group of undertakings, following agreement between the central management and a special negotiating body.

Key words: European Works Council, agreement, negotiation, procedure in Community-scale undertakings. 


\section{INTRODUCCIÓN}

La integración de España en la Unión Europea conlleva la necesidad de adaptación también del Derecho Colectivo y sus agentes a unas variables supranacionales. Es preciso, además, que la organización obrera revalorice la actividad de representación en la empresa a todos los niveles ${ }^{1}$. Tal proceder no constituye en modo alguno una "moda pasajera, sino un movimiento duradero y bien arraigado"2.

Así, cabe observar una mayor fuerza en la acción sindical, bien en el propio centro de trabajo, bien en la totalidad de la empresa, esté donde esté, bien en el conjunto de la sociedad, y ello en virtud de las nuevas coordenadas bajo las cuales se desarrollan las relaciones laborales en el siglo XXI.

Precisamente una de ellas, tan mencionada últimamente, la globalización (entendida como "internacionalización a escala planetaria del sistema económico capitalista"3) acaba siendo otro de los campos de batalla del sindicalismo actual, quizá el más importante, en tanto resalta los elementos más críticos e introduce la dimensión mundial como escenario muchas veces exasperante de los intereses contrapuestos en el interior de la fuerza de trabajo, haciendo más notoria la propia crisis de su función de representación a escala universal ${ }^{4}$.

Las organizaciones obreras se enfrentan así a la necesidad de reorientar su actividad y reordenar su estructura interna ${ }^{5}$ con el fin de adaptarse a esta nueva situación;

J. Cruz Villalón (1992) La representación de los trabajadores en la empresa y en el grupo. Sevilla: Trotta, p. 18. Además, esta participación fomenta la democracia industrial, J.M. Galiana Moreno y B. García Romero (2003) "La participación y representación de los trabajadores en la empresa en el modelo normativo español", Revista del Ministerio de Trabajo y Asuntos Sociales, 43 , p. 13 y ss.

2 M. Rodríguez-Piñero y Bravo-Ferrer (1993) "La representación de los trabajadores a nivel de empresa", Relaciones Laborales, 2, p. 2.

3 A. Baylos Grau (1999) "Globalización y Derecho del Trabajo: realidad y proyecto", Cuadernos de Relaciones Laborales, 15, p. 20.

4 A. Baylos Grau (2001) "Representación y representatividad sindical en la globalización", Cuadernos de Relaciones Laborales, 19, p. 74 y D. Lantarón Barquín (2000) "Reflexiones en torno al devenir del sindicato y a los factores que influyen en su evolución: ¿crisis y/o metamorfosis?", Revista de Derecho Social, 10, p. 72.

5 J.R. Mercader Uguina (2003) "Sistema de fuentes y globalización", Revista Española de Derecho del Trabajo, 119, p. 681. 
deben asumir nuevas responsabilidades y competencias que se salen del marco tradicional de la empresa, en un extremo, y del Estado nacional, en el otro, para adquirir una dimensión regional y sociopolítica, abierta a la colaboración con otras agrupaciones sociales y capaz de establecer mecanismos eficaces de intervención a escala internacional ${ }^{6}$.

Los sindicatos han de generar un mensaje capaz de abrir nuevos espacios de convivencia democrática, que permita recuperar la confianza de los trabajadores en torno a la posibilidad de construir un futuro basado en la justicia y la igualdad ${ }^{7}$, pues la eficacia en la actuación va a depender de su capacidad para complementar la acción empresarial y nacional con la actividad y la presión en el ámbito político internacional ${ }^{8}$.

Respondiendo a estas exigencias, el objetivo de la Directiva 94/45/CE del Consejo, de 22 de septiembre, traspuesta por la Ley 10/1997, de 24 de abril, radica en "mejorar" los derechos de información y consulta de los representantes ${ }^{9}$; en sus propias palabras:

considerando que los procedimientos de información y consulta a los trabajadores previstos en las legislaciones o prácticas de los Estados miembros no se adaptan con frecuencia a la estructura transnacional de la entidad que adopta la decisión que afecta a dichos trabajadores; que esta situación puede dar lugar a un trato desigual de los afectados por las decisiones dentro de una misma

6 S. Rodríguez Escanciano (2002) "Nuevos marcos para la acción colectiva: la implantación de un sistema de relaciones laborales más dinámico y participativo", Revista de Trabajo y Seguridad Social (Centro de Estudios Financieros, 235, p. 58, remitiendo a J.I. Palacio Morena (1993) "Prólogo", D. Albers et al. (comp.) La política regional de los sindicatos europeos. Un análisis comparativo. Madrid: MTSS, p. 21.

7 C. de la Serna Arenillas (2003) "Retos sindicales ante el siglo XXI", Revista de la Asociación Estatal de Centros Universitarios de Relaciones Laborales y Ciencias del Trabajo, 12, p. 155.

8 C. Prieto Rodríguez (2001) "Trabajo y globalización económica", Fundación Paz y Solidaridad Serafín Aliaga: Globalización, trabajo y movimiento sindical. Madrid: Fundación Paz y Solidaridad, p. 51.

$9 \quad$ M.R. Martínez Barroso (2001) "Modelos clásicos y sistemas nuevos de participación de los trabajadores en la empresa", Temas Laborales, 62, p. 63. "El desarrollo de relaciones laborales a nivel europeo ya conocía de acuerdos sobre derechos de información y consulta en empresas y grupos de empresa europeos. De hecho, la reiterada preocupación comunitaria por la promoción de los trabajadores en la vida de la empresa existía ya desde los años setenta, si bien la mejora de su posición sólo alcanzó algunos logros parciales en materias tales como: despido colectivo, transmisión de empresas, así como seguridad y salud en el trabajo", C. Sáez Lara (1996) "La Directiva 94/45, sobre el Comité de Empresa Europeo y su transposición al Derecho español", Revista Española de Derecho del Trabajo, 78, pp. 620-623. 


\begin{abstract}
empresa o de un mismo grupo; deben adoptarse las disposiciones adecuadas para velar por que los trabajadores de empresas o grupos de dimensión comunitaria sean debidamente informados y consultados en caso de que las decisiones que les afecten sean adoptadas en un Estado miembro distinto de aquél donde trabajan (Exposición de Motivos).
\end{abstract}

Su escenario, entonces, se torna transnacional, en el camino hacia la prosecución de un espacio jurídico común en la Unión Europea ${ }^{10}$.

Además, esta Directiva constituye uno de los logros más relevantes de la política social europea ${ }^{11}$, en tanto ha conseguido acercase a la realidad del momento en materia de participación, contenidos e incluso en el propio significado de la actuación comunitaria ${ }^{12}$, incardinándose dentro de un modelo participativo de información y consulta, y buscando el equilibrio entre la acción sindical reivindicativa y la de colaboración en las decisiones de gestión económica y social ${ }^{13}$ ). Al final, está destinada a transformar la participación de los trabajadores en una señal de identidad de las empresas y grupos europeos ${ }^{14}$.

La norma europea y el referente español persiguen como finalidad última la garantía del acceso de los representantes de los trabajadores a los verdaderos centros de decisión de las empresas y grupos de dimensión comunitaria. Este objetivo tiene su origen en la falta de adaptación del procedimiento de información y consulta en las respectivas legislaciones nacionales a las estructuras transnacionales cada vez más frecuentes entre las grandes empresas europeas, pues en este

10 M.E. Casas Baamonde (1997) "Dimensión transnacional de leyes nacionales, comités de empresa europeos y procesos y sentencias de conflictos colectivos", Relaciones Laborales, 18 , p. 28.

11 La Directiva 2002/14/CE, de 11 de marzo, sobre establecimiento de un marco general relativo a la información y la consulta de los trabajadores en la Comunidad Europea, constituye la "pieza de cierre" de los derechos de información y consulta a nivel comunitario, en cuanto tiende a fortalecer la armonización de los sistemas jurídicos nacionales, J. Baz Rodríguez (2002) Las relaciones de trabajo en la empresa de grupo. Granada: Comares, p. 332 y ss.

12 M.R. Cristóbal Roncero (2003) "El comité de empresa europeo en las empresas o grupos de empresas de dimensión comunitaria", Revista del Ministerio de Trabajo y Asuntos Sociales, 43, p. 157.

13 J.L. Monereo Pérez (1992) Los derechos de información de los representantes de los trabajadores. Madrid: Civitas, p. 32.

14 F. Durán López y C. Sáez Lara (1997) El papel de la participación en las nuevas relaciones laborales. Madrid: Civitas/Consejo Andaluz de Relaciones Laborales, p. 71 y M.E. Casas Baamonde (1993) "Subsidiariedad y flexibilidad del ordenamiento comunitario y participación de los trabajadores en empresas y grupos", Relaciones Laborales, 10, p. 59. 
nuevo ámbito de relaciones laborales han ido emergiendo distintos tipos de estructuras jurídico-organizativas que requieren el desarrollo de mecanismos efectivos de participación de los empleados ${ }^{15}$.

La creación de los comités de empresas europeos en las grandes empresas de dimensión comunitaria va a tener una relevante influencia refleja en la legislación española ${ }^{16}-\mathrm{y}$ en la de otros países como el Reino Unido ${ }^{17}$ - y no sólo sirve para potenciar las facultades de información y consulta de los representantes de los trabajadores, sino que, además, incorpora al acervo estatal interesantes definiciones, por ejemplo, el de grupo de empresas ${ }^{18}$.

Para alcanzar sus objetivos, conectan el ámbito europeo con dos unidades de negociación concretas: las empresas y los grupos de dimensión comunitaria ${ }^{19}$. De este modo, los comités de empresa europeos tienden a

evitar la dispersión de la iniciativa sindical, fragmentada en las distintas sedes de la empresa, acudiendo al "empresario unitario" y permite reaccionar frente a

15 M.R. Cristóbal Roncero (2003) "El comité de empresa europeo en las empresas o grupos de empresas de dimensión comunitaria", ed. cit., p. 160.

16 M.R. Martínez Barroso (2001) "Modelos clásicos y sistemas nuevos de participación de los trabajadores en la empresa", ed. cit., p. 63.

17 También la Directiva que crea los comités de empresa europeos ha sido acogida con gran interés, habida cuenta estos comités podrían revolucionar el sistema de relaciones industriales en el Reino Unido, basado tradicionalmente en las estructuras sindicales; también la información a nivel transeuropeo podría alimentar a los niveles inferiores y apropiados de la actividad sindical. Pese a lo que pudiera parecer, tal vía de participación no ha sido acogida con suspicacia y desconfianza por los sindicatos, antes al contrario, la medida ha sido apoyada por las organizaciones obreras británicas al entender que constituye un gran avance hacia nuevas áreas de consulta y negociación [M. Gold (1996) "Trade unions and democratic participation in the United Kingdom", G. Kesterm y H. Pinaud (eds.) Trade unions and democratic participation in Europe. A Scenario for the 21st Century. Hants: Avebury, p. 105 y ss.]. Incluso los procedimientos y obligaciones impuestas por el legislador comunitario ha llevado a considerar a algunos autores la existencia o el nacimiento de un doble canal de representación en el Reino Unido [D. Watling y J. Snook (2003) "Works council and trade unions: complementary or competitive? The case of SAGCo", Industrial Relations Journal, 34/3, p. 261], así como un derecho universal a la información y consulta [M. Terry (1999) "Systems of collective employee representation in non-union firms in the UK", Industrial Relations Journal, 30/1, p. 20 y H. Álvarez Cuesta (2007) "La participación de los trabajadores en el Reino Unido, ¿un avance de futuro o un mecanismo a olvidar?", Gobierno de la empresa y participación de los trabajadores: viejas y nuevas formas institucionales. XVII Congreso Nacional de Derecho del Trabajo y de la Seguridad Social. Madrid: MTAS].

18 A. Baylos Grau (2004) Sindicalismo y Derecho Sindical. Alicante: Bomarzo, p. 36.

19 M.R. Cristóbal Roncero: "El comité de empresa europeo en las empresas o grupos de empresas de dimensión comunitaria", ed. cit., p. 160. 
previsibles intentos de enfrentar concurrentemente los intereses de las distintas filiales de la multinacional ${ }^{20}$.

\title{
2. SUJETOS LEGITIMADOS Y RESPONSABILIDAD
}

destinadas a

\author{
Las disposiciones previstas en el caso español están
}

las empresas y grupos de empresas de dimensión comunitaria que tengan su dirección central en España, así como a los centros de trabajo de las citadas empresas y a las empresas de los referidos grupos cualquiera que sea el Estado miembro en que se encuentren situados (art. 5 Ley 10/1997).

De cumplirse el anterior requisito, los empresarios y trabajadores afectados tienen la facultad, previa una fase de negociación -vía convencional ${ }^{21}-$, de constituir un organismo específico de representación (comité de empresa europeo) o fijar como mecanismo alternativo el ejercicio de los derechos de información y consulta fuera del citado comitée 22 .

Uno de los extremos más delicados a analizar, y por ello más conflictivos, consiste en la información proporcionada por la dirección central de la empresa para constituir adecuadamente la comisión. Al

20 A. Baylos Grau (1999) "Globalización y Derecho del Trabajo: realidad y proyecto", Cuadernos de Relaciones Laborales, 15, p. 43.

21 J. Mercader Uguina (1997) "Derechos de información y consulta de los trabajadores en las empresas y grupos de empresas de dimensión comunitaria", Relaciones Laborales, T. II, p. 1030.

22 Este mismo esquema, en líneas generales, es el seguido por las Directivas 2001/86/CE, de 8 de octubre de 2001, por la que se completa el Estatuto de la Sociedad Anónima Europea y 2003/72/CE, de 22 de julio de 2003. por la que se completa el Estatuto de la sociedad cooperativa europea, B. Fernández Docampo (2006) La participación de los trabajadores en el Derecho Social Comunitario. Valencia: Tirant lo Blanch, p. 138. Varios han sido los análisis efectuados respecto a la primera; por citar algunos de los más relevantes, E. Garrido Pérez (2003) "La Sociedad Europea: un nuevo impulso y una nueva posibilidad para la participación de los trabajadores en las empresas", A. Grau Baylos (coord.) La dimensión europea y transnacional de la autonomía colectiva, Albacete: Bomarzo, p. 233 y ss.; F. Valdés Dal-Re (2004) "La implicación de los trabajadores en la Sociedad Europea (evolución, marco general y disposiciones de referencia)" y M.E. Casas Baamonde (2004) "La implicación de los trabajadores en la Sociedad Anónima Europea (procedimiento de negociación colectiva y diferentes modelos de implicación convenida)", ambos en G. Esteban Velasco y L. Fernández del Pozo (coords.) La Sociedad Anónima Europea. Régimen jurídico societario, laboral y fiscal. Madrid: Pons, p. 916 y 1073, respectivamente. 
respecto, el Tribunal de Justicia de la Unión Europea ya ha determinado cómo, cuando los datos sobre la estructura o la organización del grupo de empresas formen parte de la información imprescindible para iniciar negociaciones con el objeto de crear un comité de empresa europeo o de establecer un procedimiento de información y consulta transnacional de los trabajadores, corresponderá a cualquier empresa de ese grupo proporcionar los datos de que disponga, o que pueda obtener, a los órganos internos de representación de los operarios que lo soliciten ${ }^{23}$.

El legislador, consciente del problema, atribuye la responsabilidad del procedimiento negociador a la dirección central, en tanto ostenta, de conformidad con las normas legales, "la responsabilidad de establecer las condiciones y medios necesarios para la constitución de un comité de empresa europeo o el establecimiento de un procedimiento alternativo de información y consulta a los trabajadores" (art. 6).

Pero, además,

con el fin de garantizar el efecto útil de la Directiva, las demás empresas pertenecientes al grupo situadas en los Estados miembros tienen la obligación de ayudar a la dirección central presunta a cumplir la obligación principal prevista en el artículo 4.1 de la Directiva. El derecho de la dirección central presunta a recibir la información imprescindible tiene como corolario la existencia de una obligación de las direcciones de las demás empresas pertenecientes al grupo de proporcionarle la información de que dispongan o que puedan obtener... De la finalidad y de la sistemática de la Directiva se desprende que las obligaciones que incumben a la dirección central o a la dirección central presunta deben interpretarse en el sentido de que comprenden tanto la obligación de proporcionar directamente a los representantes de los trabajadores la información imprescindible para el inicio de negociaciones con el objeto de constituir un comité de empresa europeo como la de transmitir dicha información a los representantes de los trabajadores a través de la empresa perteneciente al grupo en la que trabajan, a la que dichos representantes hayan solicitado la información en primer lugar ${ }^{24}$.

23 STJCE C-62/99, de 29 de marzo de 2001, asunto Betriebsrat der bofrost Josef Boquoi Deutschland West GMBH \& Co. KG, citada por M.R. Martínez Barroso (2001) "Modelos clásicos y sistemas nuevos de participación de los trabajadores en la empresa", ed. cit., p. 64.

Anker GMBH.

24 STJCE C-349/01, de 15 de julio de 2004, asunto Betriebsrat der Firma ADS 


\section{FORMACIÓN DE LA COMISIÓN NEGOCIADORA}

El eje central que vertebra la Directiva es la autonomía de la voluntad, la cual se lleva a todas las cuestiones reguladas, desde la iniciación de los distintos procedimientos, hasta sus funciones, su composición o el alcance del derecho de información. Esta autonomía, que pretende ser respetuosa con los intereses y campo de actuación de los sujetos en presencia, constituye su mayor atractivo para quienes no son favorables a fomentar las instancias más altas de participación, haciendo posible eludir su creación ${ }^{25}$, o, aun creadas, llevarlas a una actuación ineficiente: algunas voces apuntan la posibilidad de que, en un caso extremo, el comité de empresa europeo -o el equivalente instrumento de participación y consulta-, pudiera no existir cuando los trabajadores y la dirección central de la empresa se pusieran de acuerdo para no constituirlo, sin que en tal caso resulte exigible, en la medida en que lo establecido por la Directiva no es que las partes lleguen al acuerdo de constituirlo, sino que inicien las negociaciones; y en el caso de constituirlo, es posible que sus funciones y su composición puedan tener un alcance distinto al de otras empresas o grupos o al del propio comité contemplado por la Directiva, admitiendo una diversidad que merma su eficacia ${ }^{26}$.

El procedimiento se inicia a partir de una petición de negociación (art. 7 Ley 10/1997) por parte de los sujetos legitimados ${ }^{27}$ a través de la cual formar una comisión para conseguir un acuerdo que determine la fórmula adecuada para el ejercicio de los derechos reseñados.

25 P. Menéndez Sebastián (2005) "La participación de los trabajadores en la empresa", J. García Murcia (coord.) La transposición del derecho social comunitario al ordenamiento español: un balance en el XX aniversario de la incorporación de España a la Comunidad Europea. Madrid: Ministerio de Trabajo y Asuntos Sociales, p. 529.

26 M.C. Ortiz Lallana (2007) "La participación de los trabajadores en el ámbito internacional y comunitario", Gobierno de la empresa y participación de los trabajadores: viejas y nuevas formas institucionales. XVII Congreso Nacional de Derecho del Trabajo y de la Seguridad Social. Madrid: MTAS, p. 108 y 109.

27 El artículo 6 Ley 10/1997 es de gran relevancia, en tanto en cuanto identifica al sujeto legitimado para negociar por parte de la empresa o del grupo la constitución del comité de empresa europeo o del procedimiento alternativo de información y consulta, M.R. Cristóbal Roncero (2003) "El comité de empresa europeo en las empresas o grupos de empresas de dimensión comunitaria", ed. cit., p. 164; al respecto y por extenso, J. Cabeza Pereiro (1997) "La constitución del comité de empresa europeo en una empresa o en un grupo con dirección central en España", Aranzadi Social, 5 , p. 520 y ss. 
En cuanto a la formación de esa comisión negociadora, la Directiva deja amplio margen a los Estados, habida cuenta "cada Estado miembro determinará la forma de elegir o designar a los miembros de la comisión negociadora que hayan de ser elegidos o designados en su territorio"; no obstante,

deberán prever que los trabajadores de las empresas y/o establecimientos en los que no existan representantes de los trabajadores por motivos ajenos a su voluntad tengan derecho a elegir o designar miembros de la Comisión negociadora (art. 5 Directiva 94/45/CE).

Se trata, así, de evitar que las empresas de dimensión comunitaria pretendan eludir la constitución del comité de empresa europeo o del procedimiento de información y consulta, reduciendo los trabajadores contratados por establecimiento, multiplicando los centros de reducidas dimensiones o creando la comisión negociadora sobre la base de la decisión de sólo determinados lugares ${ }^{28}$.

La concreta composición en el Derecho español está contemplada en el artículo 9 Ley 10/1997, a saber, un miembro en representación de los trabajadores de cada Estado miembro en el que la empresa de dimensión comunitaria tenga uno o más centros de trabajo o en el que se halle situada la empresa que ejerce el control de un grupo de empresas de dimensión comunitaria o una o más de las empresas controladas; en su caso, un número de miembros suplementarios en representación de los trabajadores de aquellos Estados miembros donde se hallen empleados porcentajes significativos del total de trabajadores de la empresa o grupo, de acuerdo con las siguientes reglas: un miembro en representación de los trabajadores de cada Estado miembro en que se hallen empleados desde el $25 \%$ hasta el $50 \%$ del total de trabajadores de la empresa o grupo; dos miembros en representación de los trabajadores del Estado miembro en que se hallen empleados más del $50 \%$ y hasta el $75 \%$ del total de trabajadores de la empresa o grupo; tres miembros en representación de los trabajadores del Estado miembro en que se hallen empleados más del $75 \%$ del total de trabajadores de la empresa o grupo.

64, p. 52.

28 I. Albiol Montesinos (1996) "El Comité de Empresa Europeo", Tribuna Social, 
Incluso pueden estar presentes, en las reuniones de la comisión negociadora y en las que ésta celebre con la dirección central, con voz pero sin voto, representantes elegidos por los trabajadores de Estados no miembros donde la empresa o el grupo tengan centros de trabajos o empresas, cuando así lo decidan de común acuerdo la dirección central y la comisión negociadora.

Una vez constituida, la distribución de los puestos en el seno de la comisión se hace de modo proporcional, tomando como referencia la dimensión del centro o empresa y apartándose del criterio de proporcionalidad sindical más asentado en nuestro sistema de relaciones laborales ${ }^{29}$.

Los gastos derivados del funcionamiento de la comisión serán sufragados por la dirección central, que deberá proporcionarle los recursos financieros y materiales necesarios para cumplir sus funciones adecuadamente. En particular, los siguientes: los derivados de la elección o designación de los miembros de la comisión; los de organización de las reuniones, incluidos los gastos de interpretación, manutención, alojamiento y viaje de sus miembros (los cuales, dependiendo de la ubicación de los centros en los distintos países, pueden y van a ser cuantiosos); y los derivados de un experto designado por la comisión para asistirle en sus funciones.

Si las negociaciones concluyen positivamente, la opción preferente para el legislador europeo consiste en la creación de un comité de empresa europeo, incluyendo la facultad de los negociadores de adherirse al procedimiento subsidiario previsto en el Capítulo II de la Ley $^{30}$.

En caso de resultado negativo, dichas "prolijas"31 disposiciones subsidiarias serán de aplicación obligatoria, constituyendo tal opción la

29 J. Mercader Uguina (1997) "Derechos de información y consulta de los trabajadores en las empresas y grupos de empresas de dimensión comunitaria", ed. cit., p. 1031 y M.R. Cristóbal Roncero (2003) "El comité de empresa europeo en las empresas o grupos de empresas de dimensión comunitaria", ed. cit., p. 167.

30 B. Fernández Docampo (2006) La participación de los trabajadores en el Derecho Social Comunitario, ed. cit., p. 178 y ss.

31 M.R. Cristóbal Roncero (2003) "El comité de empresa europeo en las empresas o grupos de empresas de dimensión comunitaria", ed. cit., p. 171. 
"vía imperativa"32, aun cuando su finalidad última radique en la promoción del establecimiento convencional de las instancias representativas ${ }^{33}$.

\section{EUROPEO}

\section{RESULTADO DE LA NEGOCIACIÓN: EL COMITÉ DE EMPRESA} negociadora deberá contener:

El acuerdo entre la dirección central y la comisión

- la identificación de las partes que lo conciertan;

- la determinación de los centros de trabajo de la empresa de dimensión comunitaria o de las empresas del grupo de empresas de dimensión comunitaria afectados por el acuerdo;

- la composición del comité de empresa europeo, el número de sus miembros, su distribución y la duración de su mandato, así como los efectos que sobre ello se deriven de las modificaciones en la estructura de la empresa o grupo o en la composición de los órganos nacionales de representación de los trabajadores;

- las atribuciones del comité de empresa europeo y el procedimiento de información y consulta al mismo;

- el lugar, la frecuencia y la duración de las reuniones del comité de empresa europeo;

- los recursos materiales y financieros asignados al comité de empresa europeo para el adecuado cumplimiento de sus funciones; $y$,

- la duración del acuerdo y las condiciones de su denuncia, prórroga y renegociación.

32 J. Mercader Uguina (1997) "Derechos de información y consulta de los trabajadores en las empresas y grupos de empresas de dimensión comunitaria", ed. cit., p. 1034.

33 M.R. Cristóbal Roncero (2003) "El comité de empresa europeo en las empresas o grupos de empresas de dimensión comunitaria", ed. cit., p. 172; J. Mercader Uguina (1997) "Derechos de información y consulta de los trabajadores en las empresas y grupos de empresas de dimensión comunitaria", ed. cit., p. 1035; J.M. Ramirez Martínez (1996) "Perspectivas del derecho social en la Comunidad Europea (con especial atención al desarrollo de la Directiva sobre Comité de empresa europeo)", Tribuna Social, 67, p. 16 y ss.; J. Baz Rodríguez (1998) "El sistema europeo de información y consulta de los trabajadores en las empresas y grupos de dimensión comunitaria: reflexiones en torno a la Ley 10/1997, de 24 de abril", Relaciones Laborales, 3, p. 268. 
La composición del comité es un asunto de indudable trascendencia, tanto para los sindicatos como para la representación unitaria en España; pero es uno de los puntos sobre los que la Directiva guarda "absoluto mutismo" 34 , sólo dispone que, a falta de acuerdo,

estará compuesto por trabajadores de la empresa o grupo, elegidos o designados por y entre los representantes de los trabajadores o, en su defecto, por el conjunto de los trabajadores, de conformidad con las legislaciones y prácticas nacionales (art. 17.1 Ley 10/1997, traspuesto de forma literal de la Directiva 94/45/CE).

El legislador prescinde de organizaciones de ámbito más amplio (confederaciones sindicales europeas) y remite a las legislaciones o prácticas nacionales la elección o designación de los mismos.

En consecuencia, las legislaciones de cada Estado miembro deberán realizar remisiones entre ellas, de modo que los órganos de aplicación de las normas de cada uno de ellos tendrán que hacer uso frecuente de los preceptos correspondientes a los demás, manteniendo, tanto la Directiva 94/45/CE, como la Ley $10 / 1997$, una postura neutral sobre el carácter de los representantes que participen en la comisión negociadora, ya que se remiten a procedimientos de elección o designación ${ }^{35}$. Con ello se busca facilitar y reconocer la posibilidad de elección a todos los trabajadores de todos los Estados miembros, incluso en aquellos casos en los cuales no exista "un sistema generalizado de representación" ${ }^{36}$ como sucede en Irlanda y Gran Bretaña ${ }^{37}$.

En España, la Ley 10/1997 encomienda tal función de forma indistinta tanto a la representación sindical como a la unitaria, exigiendo a ambas probar su representatividad:

los representantes que deban formar parte de la comisión negociadora y del comité de empresa europeo serán designados por acuerdo de aquellas representaciones

34 B. Fernández Docampo (2006) La participación de los trabajadores en el Derecho Social Comunitario, ed. cit., p. 183.

35 J. Cabeza Pereiro (1997) "La constitución del comité de empresa europeo en una empresa o en un grupo con dirección central en España", ed. cit., p. 527.

36 F. Durán López y C. Sáez Lara (1997) El papel de la participación en las nuevas relaciones laborales, ed. cit., p. 91.

37 B. Fernández Docampo (2006) La participación de los trabajadores en el Derecho Social Comunitario, ed. cit., p. 184. 
sindicales que en su conjunto sumen la mayoría de los miembros del comité o comités de empresa y delegados de personal en su caso, o por acuerdo mayoritario de dichos miembros y delegados

debiendo ser el trabajador elegido delegado de personal, miembro del comité de empresa o delegado sindical (art. 27). La norma vuelve a hablar de mayoría y no de proporcionalidad y así ha sido corroborado por los Tribunales ${ }^{38}$.

En raras ocasiones, y la práctica así parece corroborarlo, van a cumplir este destacado papel los representantes unitarios, la mayoría de las veces serán los sindicatos a través de sus delegados quienes asuman esta competencia.

No obstante, el comité creado al amparo del artículo 12 Ley 10/1997 podrá ofrecer un régimen de elección distinto e incluso ampliar el círculo de sujetos capaces de ostentar ese cargo representativo, pudiendo recaer en terceras personas sin relación laboral con la empresa o grupo ${ }^{39}$. A estos representantes a nivel europeo, el legislador -junto a las garantías ya disfrutadas por su calidad de representantes unitarios o sindicales ${ }^{40}$ - les concede permiso retribuido para acudir a las reuniones y un crédito de horas adicional de sesenta horas anuales (art. 28 Ley 10/1997). Tal enunciado permite deducir que las reuniones del comité de empresa europeo así como las previas a la reunión con la dirección central no consumen el crédito horario "europeo", así como la imposibilidad de acumular éste en uno sólo de los representantes españoles.

La otra cara de la moneda viene constituida por un deber de confidencialidad (el sigilo y el secreto) en esta sede específicamente consignado y con mayor intensidad que en el $\mathrm{ET}^{41}$ :

los miembros de la comisión negociadora y del comité de empresa europeo y los representantes de los trabajadores en el marco de un procedimiento alternativo de información y consulta, así como los expertos que les

38 SAN 25 junio 2007 (Jur. 246353).

39 N. Mendoza Navas (2003) "La construcción de instrumentos de representación a nivel transnacional: el Comité de Empresa Europeo y la experiencia española", A. Baylos Grau (coord.) La dimensión europea y transnacional de la autonomía colectiva. Albacete: Bomarzo, p. 142.

40 Aplicando ambas, SAN 10 julio 2001 (Jur. 308102).

41 M.R. Cristóbal Roncero (2003) "El comité de empresa europeo en las empresas o grupos de empresas de dimensión comunitaria", ed. cit., p. 175. 


\begin{abstract}
asistan, no estarán autorizados a revelar a terceros aquella información que les haya sido expresamente comunicada a título confidencial. Esta obligación de confidencialidad subsistirá incluso tras la expiración de su mandato e independientemente del lugar en que se encuentren. Excepcionalmente, la dirección central no estará obligada a comunicar aquellas informaciones específicas relacionadas con secretos industriales, financieros 0 comerciales cuya divulgación pudiera, según criterios objetivos, obstaculizar el funcionamiento de la empresa u ocasionar graves perjuicios en su estabilidad económica. Esta excepción no abarca aquellos datos que tengan relación con el volumen de empleo en la empresa (art. 22 Ley 10/1997).
\end{abstract}

Este mecanismo adicional trata de garantizar la posibilidad de la empresa de establecer una reserva absoluta sobre determinadas informaciones que abarcan desde la "información tecnológica" (procedimientos de fabricación, reparación, características de rendimiento de la maquinaria, etc.) hasta "información no tecnológica" (marketing, organización interna de la empresa con sus clientes y proveedores, lista de clientes, etc.) cuya posible divulgación pudiera provocar en la empresa un temor razonable a la competencia desleal por parte de otras o a la hipotética repercusión en su prestigio empresarial, etc.; en definitiva, la norma bebe del tradicional concepto de secreto empresarial que englobaría las distintas clases ya mencionadas ${ }^{42}$.

No obstante, la dirección central y la comisión negociadora podrán elegir otra vía y acordar, en vez de la constitución de un comité de empresa europeo, el establecimiento de uno o más procedimientos de información y consulta a los trabajadores sobre aquellas cuestiones transnacionales que puedan afectar considerablemente a sus intereses. En tal caso, el acuerdo deberá prever las modalidades con arreglo a las cuales los representantes de los trabajadores tendrán derecho a reunirse para deliberar acerca de la información que les sea comunicada (art. 12.2 Ley 10/1997).

42 Idem 


\section{COMPETENCIAS DEL COMITÉ DE EMPRESA EUROPEO}

Una vez formado conviene examinar sus competencias y, vinculados con éstas, los derechos de información que ostenta el comité.

En caso de acuerdo entre los sujetos negociadores, las partes negociadoras establecerán libremente el listado de materias sobre las que serán informados y consultados los integrantes de la instancia representativa transnacional y la intensidad de la interlocución, así como sobre el procedimiento y particularidades de los mecanismos previstos ${ }^{43}$.

En cambio, de acogerse a la regulación subsidiaria, y sin perjuicio de otras cuestiones que puedan plantearse, en la reunión anual se analizarán aquéllas relacionadas con la estructura de la empresa, su situación económica y financiera, la evolución probable de las actividades, la producción y las ventas, la situación y evolución probable del empleo, las inversiones, los cambios sustanciales que afecten a la organización, la introducción de nuevos métodos de trabajo o de producción, los traslados de producción, las fusiones, la reducción del tamaño o el cierre de empresas, centros de trabajo o partes importantes de éstos, y los despidos colectivos...; [además,] deberá ser informado con la debida antelación de aquellas circunstancias excepcionales que afecten considerablemente a los intereses de los trabajadores, especialmente en los casos de traslados de empresas, de cierres de centros de trabajo o empresas o de despidos colectivos (art. 18.3 Ley 10/1997).

Tal lista es únicamente orientativa, pues otras situaciones conflictivas transnacionales también requerirán la participación del comité ${ }^{44}$.

43 J. Baz Rodríguez (1998) "El sistema europeo de información y consulta de los trabajadores en las empresas y grupos de dimensión comunitaria: reflexiones en torno a la Ley 10/1997, de 24 de abril", Relaciones Laborales, 3, p. 263.

44 N. Mendoza Navas (2003) "La construcción de instrumentos de representación a nivel transnacional: el Comité de Empresa Europeo y la experiencia española", ed. cit., p. 145. 


\section{EL COMITÉ DE EMPRESA EUROPEO EN LAS EMPRESAS}

\section{ESPAÑOLAS}

Analizando la aplicación de la ley en la práctica empresarial española, los resultados, si no muy halagüeños, sí dejan entrever un resquicio para la esperanza, siquiera sea en pos de una buena publicidad de la marca.

Los acuerdos por los que se crean comités de empresa europeos ratificados en España son escasos, alrededor de la treintena. Esta apatía ha sido justificada aludiendo al pequeño número de grandes empresas españolas con implantación en Europa, el bajo nivel de implantación sindical en las mismas, la inexistencia de una negociación colectiva centralizada, la generalización de la subcontratación, la diversificación de actividades de la empresa central ${ }^{45}$...; sin dejar de lado tampoco las dificultades provocadas por la diversidad idiomática ${ }^{46}$ y la excesiva identificación con los intereses o problemas del centro del trabajo del país al que representan ${ }^{47}$.

Las empresas que en España han formado un comité de empresa a este nivel han optado por atribuirle competencias económicas y sociales de carácter estratégico y trasnacional a nivel del grupo, fundamentalmente en temas relacionados con la situación económica, financiera y social, la organización, la seguridad y salud en el trabajo y el desarrollo sostenible ${ }^{48}$, excluyendo otras (el comité no será competente

45 C. Piqueras Piqueras (2003) "Análisis de algunos supuestos de creación por la negociación colectiva de órganos de representación de los trabajadores en empresas y grupos de empresas de dimensión comunitaria en España", A. Baylos Grau (coord.) La dimensión europea y transnacional de la autonomía colectiva. Albacete: Bomarzo, p. 207, citando un Informe elaborado por la Confederación Sindical de CC.OO.

46 Por tal motivo, los acuerdos negociados prevén, para un desarrollo eficaz de las reuniones, o bien la traducción simultánea [Acuerdos GENERAL CABLE; REPSOL-YPF; ALTADIS y ROCA], o bien la asistencia de intérpretes [Acuerdos POWER CONTROL y PRAXAIR].

47 C. Piqueras Piqueras (2003) "Análisis de algunos supuestos de creación por la negociación colectiva de órganos de representación de los trabajadores en empresas y grupos de empresas de dimensión comunitaria en España", ed. cit., p. 208.

48 Comité de empresa europeo del Grupo Arcelor, en http://www.arcelor.com/ index.php?lang=es\&page=65. "En la reunión general anual se tratarán de cuestiones que afecten al conjunto de las empresas que integran el Grupo BBVA representadas en la reunión y que tengan interés general, relacionadas con aspectos tales como: estructura de Empresa, su situación económica y financiera, evolución probable de las actividades, la producción y las ventas, la situación y evolución probable del empleo, las inversiones, los cambios sustanciales que afecten a la organización, la introducción de nuevos métodos de trabajo, los traslados de producción, las fusiones, la reducción del tamaño o el cierre de empresas, centros de trabajo, y los despidos colectivos. Asimismo el Comité de Empresa tendrá derecho a ser informado con la debida antelación sobre 
ni tratará cuestiones locales o nacionales sujetas a la legislación o convenios colectivos nacionales o aspectos como la jornada de trabajo, salarios $\mathrm{u}$ otros beneficios, o asuntos relativos a empleados individuales, que deberán ser tratados dentro de las estructuras, normativas y procedimientos aplicados en cada filial del grupo ${ }^{49}$ ).

Más recientemente y siguiendo la estela, tanto en su estructura como en los mecanismos de participación en las comisiones, marcada por la Directiva y posterior Ley sobre comités de empresa europeos, ha entrado en vigor la Ley 31/2006, de 18 de octubre, sobre implicación de los trabajadores en las sociedades anónimas y cooperativas europeas, trasponiendo la Directiva 2001/86/CE, del Consejo, de 8 de octubre de 2001, por la que se completa el Estatuto de la Sociedad Anónima Europea en lo que respecta a la implicación de los trabajadores. De nuevo esta norma diseña un procedimiento negocial entre representantes de los trabajadores y empresario por medio del cual pacten las vías de participación en la corporación; a falta de acuerdo ad hoc o por decisión de las partes, entrarán en juego las normas subsidiarias previstas en el Capítulo II.

En fin, es menester invocar a Cronos para que, a modo de Juez supremo, determine la efectividad de esta última norma en orden a mejorar la participación de los trabajadores en este tipo de empresas $\mathrm{y}$, en último término, contribuir a mejorar las condiciones laborales a nivel europeo.

\section{BIBLIOGRAFÍA}

Albiol MONTESINOS, I. (1996) "El Comité de Empresa Europeo", Tribuna Social, 64, pp. 47-58.

Álvarez CUeSTA, H. (2007) "La participación de los trabajadores en el Reino Unido, ¿un avance de futuro o un mecanismo a olvidar?. Gobierno de la

aquellas circunstancias excepcionales que afecten considerablemente a los intereses de los trabajadores, especialmente en los casos de traslados de empresas, de cierres de centros de trabajo o empresas o despidos colectivos", Acuerdo para la Constitución de Comité de Empresa Europeo del grupo BBVA, en http: / / www.comfia. net/territorio. php?num=18\&modo=leer\&art=1082.

49 Comité de empresa europeo del Grupo Santander, en http://www.lukor.com/ not-neg/laboral/0505/30112710.htm. 
empresa y participación de los trabajadores: viejas y nuevas formas institucionales. XVII Congreso Nacional de Derecho del Trabajo y de la Seguridad Social. Madrid: MTAS, pp. 1-215.

BAYLOS GRAU, A. (1999) "Globalización y Derecho del Trabajo: realidad y proyecto", Cuadernos de Relaciones Laborales, 15, pp. 19-49.

- (2001) "Representación y representatividad sindical en la globalización", Cuadernos de Relaciones Laborales, 19, pp. 69-94.

- (2004) Sindicalismo y Derecho Sindical. Alicante: Bomarzo.

BAZ RODRíGUEZ, J. (1998) "El sistema europeo de información y consulta de los trabajadores en las empresas y grupos de dimensión comunitaria: reflexiones en torno a la Ley 10/1997, de 24 de abril", Relaciones Laborales, 3, pp. 250-278.

- (2002) Las relaciones de trabajo en la empresa de grupo. Granada: Comares.

Cabeza Pereiro, J. (1997) "La constitución del comité de empresa europeo en una empresa o en un grupo con dirección central en España", Aranzadi Social, 5, pp. 515-544.

CASAS BAAMONDE, M.E. (1993) "Subsidiariedad y flexibilidad del ordenamiento comunitario y participación de los trabajadores en empresas y grupos", Relaciones Laborales, 10, pp. 59-70.

- (1997) "Dimensión transnacional de leyes nacionales, comités de empresa europeos y procesos y sentencias de conflictos colectivos", Relaciones Laborales, 18, pp. 27-41.

- (2004) "La implicación de los trabajadores en la Sociedad Anónima Europea (procedimiento de negociación colectiva y diferentes modelos de implicación convenida)". L. FernándeZ DEL Pozo y G. Esteban VeLASCO (coords.) La Sociedad Anónima Europea: Régimen jurídico societario, laboral y fiscal. Madrid: Pons, pp. 996-1071.

CRISTÓBAL RONCERO, M.R. (2003) "El comité de empresa europeo en las empresas o grupos de empresas de dimensión comunitaria", Revista del Ministerio de Trabajo y Asuntos Sociales, 43, pp. 157-180.

CRUZ VILLALÓN, J. (1992) La representación de los trabajadores en la empresa y en el grupo. Sevilla: Trotta.

DURÁN LóPEZ, F. y C. SÁEZ LARA (1997) El papel de la participación en las nuevas relaciones laborales. Madrid: Civitas/Consejo Andaluz de Relaciones Laborales.

FERNÁNDEZ DOCAMPO, B. (2006) La participación de los trabajadores en el Derecho Social Comunitario. Valencia: Tirant lo Blanch. 
Galiana Moreno, J.M. y B. García Romero (2003) "La participación y representación de los trabajadores en la empresa en el modelo normativo español", Revista del Ministerio de Trabajo y Asuntos Sociales, 43, pp. 13-30.

GaRRIDo PÉreZ, E. (2003) "La Sociedad Europea: un nuevo impulso y una nueva posibilidad para la participación de los trabajadores en las empresas". A. BAYLOS GRAU (coord.) La dimensión europea y transnacional de la autonomía colectiva. Albacete: Bomarzo, pp. 233-270.

GoLD, M. (1996) "Trade unions and democratic participation in the United Kingdom". G. KESTERM y H. PINAUD (eds.) Trade unions and democratic participation in Europe. A Scenario for the 21st Century. Hants: Avebury, pp. 1-220.

LANTARÓN BARQUín, D. (2000) "Reflexiones en torno al devenir del sindicato y a los factores que influyen en su evolución: ¿crisis y/o metamorfosis?", Revista de Derecho Social, 10, pp. 55-80.

MARTíneZ BARROSO, M.R. (2001) "Modelos clásicos y sistemas nuevos de participación de los trabajadores en la empresa", Temas Laborales, 62, pp. 31-68.

MENDOZA NAVAS, N. (2003) "La construcción de instrumentos de representación a nivel transnacional: el Comité de Empresa Europeo y la experiencia española". A. BAYLOS GRAU (coord.) La dimensión europea y transnacional de la autonomía colectiva. Albacete: Bomarzo, pp. 131-152.

MENÉNDEZ SEBASTIÁN, P. (2005) "La participación de los trabajadores en la empresa". J. GARCÍA MURCIA (coord.) La transposición del derecho social comunitario al ordenamiento español: un balance en el XX aniversario de la incorporación de España a la Comunidad Europea. Madrid: Ministerio de Trabajo y Asuntos Sociales, pp. 523-545.

MerCADER Uguina, J. (1997) "Derechos de información y consulta de los trabajadores en las empresas y grupos de empresas de dimensión comunitaria", Relaciones Laborales, T. II, pp. 1023-1044.

- (2003) "Sistema de fuentes y globalización", Revista Española de Derecho del Trabajo, 119, pp. 667-690.

MONEREO PÉREZ, J.L. (1992) Los derechos de información de los representantes de los trabajadores. Madrid: Civitas.

PAlacio MorenA, J.I. (1993) "Prólogo". D. Albers et al. (comp.) La política regional de los sindicatos europeos. Un análisis comparativo. Madrid: MTSS, pp. 1-7. 
PiQUeras PiQUeras, C. (2003) "Análisis de algunos supuestos de creación por la negociación colectiva de órganos de representación de los trabajadores en empresas y grupos de empresas de dimensión comunitaria en España". A. BAYLOS GRAU (coord.) La dimensión europea y transnacional de la autonomía colectiva. Albacete: Bomarzo, pp. 1-250.

PRIETo RodRíGUEZ, C. (2001) "Trabajo y globalización económica". Fundación Paz y Solidaridad Serafín Aliaga: Globalización, trabajo y movimiento sindical. Madrid: Fundación Paz y Solidaridad, pp. 1-110.

Ramírez MARTínez, J.M. (1996) "Perspectivas del derecho social en la Comunidad Europea (con especial atención al desarrollo de la Directiva sobre Comité de empresa europeo)", Tribuna Social, 67, pp. 9-19.

RODRíGUEZ ESCANCIANO, S. (2002) "Nuevos marcos para la acción colectiva: la implantación de un sistema de relaciones laborales más dinámico y participativo", Revista de Trabajo y Seguridad Social (Centro de Estudios Financieros), 235, pp. 3-60.

RODRÍGUEZ-PIÑERO Y BRAVO-FERRER, M. (1993) "La representación de los trabajadores a nivel de empresa", Relaciones Laborales, 2, pp. 12-18.

SÁEZ LARA, C. (1996) "La Directiva 94/45, sobre el Comité de Empresa Europeo y su transposición al Derecho español", Revista Española de Derecho del Trabajo, 78, pp. 20-45.

Serna ARENILLAS, C. de la (2003) "Retos sindicales ante el siglo XXI", Revista de la Asociación Estatal de Centros Universitarios de Relaciones Laborales y Ciencias del Trabajo, 12, pp. 149-160.

TERRY, M. (1999) "Systems of collective employee representation in non-union firms in the UK", Industrial Relations Journal, 30/1, pp. 12-78.

VALDÉS DAL-RE, F. (2004) "La implicación de los trabajadores en la Sociedad Europea (evolución, marco general y disposiciones de referencia)". G. Esteban Velasco y L. FeRnándeZ del POZO (coords.) La Sociedad Anónima Europea. Régimen jurídico societario, laboral y fiscal. Madrid: Pons, pp. $1072-1111$.

WATLING, D. y J. SNOOK (2003) "Works council and trade unions: complementary or competitive? The case of SAGCo", Industrial Relations Journal, 34/3, pp. 45-86. 